\title{
MAP OF QUASICOMPONENTS INDUCED BY A SHAPE MORPHISM
}

\author{
Nikita Shekutkovski, Tatjana Atanasova-Pachemska and GJorgui \\ MARKOSKI
}

Ss. Cyril and Methodius University and University "Goce Delchev" - Shtip, Republic of Macedonia

\begin{abstract}
Using the intrinsic definition of shape we prove an analogue of well known Borsuks theorem for compact metric spaces.

Suppose $X$ and $Y$ are locally compact metric spaces with compact spaces of quasicomponents $Q X$ and $Q Y$. For a shape morphism $f: X \rightarrow Y$ there exists a unique continuous map $f_{\#}: Q X \rightarrow Q Y$, such that for a quasicomponent $Q$ from $X$ and $W$ a clopen set containing $f_{\#}(Q)$ the restriction $f: Q \rightarrow W$, is a shape morphism, also.
\end{abstract}

\section{INTRODUCTION}

This is the well known theorem of Borsuk about the set of components $\square X$ of a compact metric space $X$.

Theorem 1.1 (Borsuk). Let $X$ and $Y$ be compact metric spaces. Then for any approximative map $f$ from $X$ towards $Y$, there exists an unique map $f^{\wedge}: \square X \rightarrow \square Y$ such that for any component $C_{0}$ of $X$, the restriction $f_{\mid}$to $C_{0}$ is an approximative map from $C_{0}$ to $f^{\wedge}\left(C_{0}\right)$.

Moreover, if $f$ and $g$ define the same shape morphism, then $f^{\wedge}=g^{\wedge}$.

There are several generalizations of the theorem of Borsuk in non compact case. For the case of spaces with compact components see $[2-4,8,12]$ and under conditions $\square X, \square Y$ to be $\mathbb{N}$-compact spaces and projections $p: X \rightarrow \square X$, $p: Y \rightarrow \square Y$ to be closed, see [9, Corollary 1.5.].

For non compact spaces, it is expected that we have to use quasicomponents instead of components.

2010 Mathematics Subject Classification. 54C56, 55P55, 54C08.

Key words and phrases. Intrinsic definition, continuity up to a covering, proximate sequence, proximate net, quasicomponents. 
In [11], it is shown that if $S h(X)=S h(Y)$ then $\mathbb{N}$-compactifications of $Q X$ and of $Q Y$ are homeomorhic. A similar result is given in [10].

Using the intrinsic definition of shape, we give an analogue of the Borsuks theorem in the case of noncompact spaces under condition $Q X$ to be compact.

The most known approach for the intrinsic definition of shape of (metric) spaces is by use of functions $f: X \rightarrow Y$ which are near to continuous. The idea of $\varepsilon$ - continuity (continuity up to $\varepsilon>0$ ) leads to a continuity up to some covering $\mathcal{V}$ of $Y$, i.e., $\mathcal{V}$-continuity, and corresponding $\mathcal{V}$-homotopy.

Definition 1.2. Let $X, Y$, be spaces, and $\mathcal{V}$ be a covering of $Y$. A function $f: X \rightarrow Y$ is $\mathcal{V}$-continuous, if for any $x \in X$, there exists a neighborhood $U$ of $x$, such that $f(U) \subseteq V$, for some member $V \in \mathcal{V}$.

The family of all $U$, form a covering $\mathcal{U}$ of $X$. Shortly, we say that $f$ : $X \rightarrow Y$ is $\mathcal{V}$-continuous, if there exists $\mathcal{U}$ such that $f(\mathcal{U}) \prec \mathcal{V}$.

Definition 1.3. Two $\mathcal{V}$ - continuous functions $f, g: X \rightarrow Y$ are $\mathcal{V}$ homotopic, if there exists a function $F: X \times I \rightarrow Y$ such that:

1) $F: X \times I \rightarrow Y$ is st $\mathcal{V}$ - continuous;

2) There exists a neighborhood $N=[0, \varepsilon) \cup(1-\varepsilon, 1]$ of $\{0,1\}$ in $[0,1]$ such that $\left.F\right|_{X \times N}$ is $\mathcal{V}$ - continuous;

3) $F(x, 0)=f(x), F(x, 1)=g(x)$.

The relation of homotopy between $\mathcal{V}$ - continuous functions is an equivalence relation.

REMARK 1.4. The conditions 1) and 2), in the previous definition cannot be replaced by a requirement $F: X \times I \rightarrow Y$ to be a $\mathcal{V}$ - continuous function, since in this case the juxtaposition of homotopies doesn't work and the relation of homotopy wouldn't be an equivalence relation. This is shown by [15, Theorem 1.1] and in the example in [1].

We mention that Akaike and Sakai in the paper [1] introduced an intrinsic definition of proper shape for locally compact separable metric spaces, which is shown to be equivalent with the usual proper shape theory [14]. Omitting the word proper in their definitions we obtain a shape theory.

Also, we ommit their additional requirement coverings to consist of open sets with compact closures, since they needed this requirement only for proper functions.

We consider the set $\operatorname{cov} X$ to be the set of all star finite coverings. The requirement is connected with the requirement of st $\mathcal{V}$-continuity of a homotopy connecting two $\mathcal{V}$-continuous functions. We mention that in the case of locally compact spaces any covering has a locally finite refinement iff any covering has a star finite refinement. So, for paracompact locally compact spaces any covering has: 1) locally finite refinement 2) star finite refinement 
(i.e both locally finite coverings and star finite coverings are cofinite in the set of all coverings) [6].

We repeat the main notions for the intrinsic definition of shape for metric locally compact spaces.

A proximate net $\left(f_{\mathcal{V}}\right): X \rightarrow Y$ is a net of functions $f_{\mathcal{V}}: X \rightarrow Y, f_{\mathcal{V}}$ is a $\mathcal{V}$-continuous function, indexed by all coverings of $\operatorname{cov} Y$, such that if $\mathcal{V} \succ \mathcal{W}$ then $f_{\mathcal{V}}$ and $f_{\mathcal{W}}$ are $\mathcal{V}$-homotopic.

Two proximate nets $\left(f_{\mathcal{V}}\right),\left(f_{\mathcal{V}}^{\prime}\right): X \rightarrow Y$ are homotopic if $f_{\mathcal{V}}$ and $f_{\mathcal{V}}^{\prime}$ are $\mathcal{V}$-homotopic, for all $\mathcal{V} \in \operatorname{cov} Y$ which we denote by $\left(f_{\mathcal{V}}\right) \sim\left(f_{\mathcal{V}}^{\prime}\right)$.

This is an equivalence relation.

If $\left(f_{\mathcal{V}}\right): X \rightarrow Y$ and $\left(g_{\mathcal{W}}\right): Y \rightarrow Z$ are proximate nets, then for a covering $\mathcal{W} \in \operatorname{cov} Z$, there exists a covering $\mathcal{V} \in \operatorname{cov} Y$ such that $g_{\mathcal{W}}(\mathcal{V}) \prec \mathcal{W}$. Then the composition of these two proximate nets is a proximate net $\left(h_{\mathcal{W}}\right)$ defined by $\left(h_{\mathcal{W}}\right)=\left(g_{\mathcal{W}} f_{\mathcal{V}}\right): X \rightarrow Z$.

Spaces and homotopy classes of proximate nets form the the category whose isomorphisms induces classification which coincide with shape classification, i.e., isomorphic spaces in this category have the same shape.

\section{QuASICOMPONENTS}

Usually, the quasicomponent of a point is defined as an intersection of all clopen (=closed and open) sets containing that point. The component of a point $x$ is contained in the quasicomponent of $x$.

Here we give another definition of quasicomponents using functional separation.

Definition 2.1. Subsets $A$ and $B$ of $X$ are said to be functionally separated in $X$ if there exists a continuous function $f: X \rightarrow\{0,1\}$ such that $f(A)=\{0\}$ and $f(B)=\{1\}$.

DEFINITION 2.2. Quasicomponent $Q$ of a point $x$ consists of all points $y$ which cannot be functionally separated from $x$.

By $Q(X)$ we denote the set of quasicomponents of $X$. We define a topology of $Q(X)$ by defining a base for this topology to be the family $Q F=\{A \mid A \in Q(X), A \subseteq F\}$, where $F$ is clopen subset of $X$. The set $Q(X)$ with this topology is the space of quasicomponents of $X$. The set $Q F$ is clopen in $Q(X)$.

THEOREM 2.3. If $\mathcal{W}$ is a covering of $Y$ consisting of disjoint open sets (shortly disjoint covering) and $f: X \rightarrow Y$ is a $\mathcal{W}$ - continuous function, then for each quasicomponent $Q$ of $X$, there exists $W_{Q} \in \mathcal{W}$ such that $f_{\mathcal{W}}(Q) \subseteq$ $W_{Q}$

Proof. Let $x \in X, Q$ is quasicomponent of $X$ and $W_{Q} \in \mathcal{W}$ such that $f(x) \in W_{Q}$. We define a continuous map $h: Y \rightarrow\{0,1\}$ by $h\left(W_{Q}\right)=\{0\}$ and $h\left(Y \backslash W_{Q}\right)=\{1\}$. 
We will prove that $h f: X \rightarrow\{0,1\}$ is continuous.

For an arbitrary point $z \in X$ there exists a neighborhood $U_{z}$ and there exists $W \in \mathcal{W}$ such that $f\left(U_{z}\right) \subseteq W$.

Now, if $W=W_{Q}$, then $h f\left(U_{z}\right) \subseteq h\left(W_{Q}\right) \subseteq\{0\}$, while if $W \neq W_{Q}$, $h f\left(U_{z}\right) \subseteq h(W) \subseteq\{1\}$. It follows that the composition $h f: X \rightarrow\{0,1\}$ is continuous.

If we suppose that there is a point $y \in Q$ such that $f(y) \notin W_{Q}$, then $x$ and $y$ will be functionally separated by the map $h f: X \rightarrow\{0,1\}$. We conclude that $f(Q) \subseteq W_{Q}$.

COROLlary 2.4. If $\mathcal{W}$ is a covering consisting of disjoint open sets and $f: X \rightarrow Y$ is a $\mathcal{W}$-continuous function, then for each component $Q \in \square X$, there exists $W_{Q} \in \mathcal{W}$ such that $f_{\mathcal{W}}(Q) \subseteq W_{Q}$.

THEOREM 2.5. If $\mathcal{W}$ is a covering consisting of disjoint open sets, and $H: X \times I \rightarrow Y$ is a $\mathcal{W}$-homotopy connecting $\mathcal{W}$ - continuous functions $f: X \rightarrow Y$ and $g: X \rightarrow Y$, then for each quasicomponent $Q$ of $X$, there exists $W_{Q} \in \mathcal{W}$ such that $f(Q) \subseteq W_{Q}$ and $g(Q) \subseteq W_{Q}$.

Proof. Since $H: X \times I \rightarrow Y$ is st $\mathcal{W}$ continuous and $\mathcal{W}=\operatorname{st} \mathcal{W}$, it follows that $H: X \times I \rightarrow Y$ is $\mathcal{W}$ - continuous. Similarly as in the previous theorem, there exists $W_{Q} \in \mathcal{W}$ such that $H(Q \times I) \subseteq W_{Q}$. Then $f(Q)=$ $H(Q \times\{0\}) \subseteq W_{Q}$ and $g(Q)=H(Q \times\{1\}) \subseteq W_{Q}$

Corollary 2.6. If $\mathcal{W}$ is a covering consisting of disjoint open sets, and $H: X \times I \rightarrow Y$ is a $\mathcal{W}$-homotopy connecting $\mathcal{W}$ - continuous functions $f: X \rightarrow Y$ and $g: X \rightarrow Y$, then for each component $Q \in \square X$, there exists $W_{Q} \in \mathcal{W}$ such that $f(Q) \subseteq W_{Q}$ and $g(Q) \subseteq W_{Q}$.

TheOREM 2.7. Let $\left(f_{\mathcal{V}}\right): X \rightarrow Y$ be a proximate net, and $\mathcal{W}$ be a covering consisting of disjoint open sets. Then, for each quasicomponent $Q$ of $X$, there exists $W_{Q} \in \mathcal{W}$ such that for every $\mathcal{V} \prec \mathcal{W}, f_{\mathcal{V}}(Q) \subseteq W_{Q}$ (and specially $\left.f_{\mathcal{W}}(Q) \subseteq W_{Q}\right)$

Proof. By Theorem 2.3, there exists a $W_{Q} \in \mathcal{W}$ such that $f_{\mathcal{W}}(Q) \subseteq$ $W_{Q}$. If $\mathcal{V} \prec \mathcal{W}$, then there exists a st $\mathcal{W}$-continuous function, $H_{\mathcal{W} \mathcal{V}}: X \times I \rightarrow$ $Y$. Since st $\mathcal{W}=\mathcal{W}, H_{\mathcal{W W}}: X \times I \rightarrow Y$ is also a $\mathcal{W}$-continuous function. Since $H_{\mathcal{W V}}(Q \times\{0\})=f_{\mathcal{W}}(Q) \subseteq W_{Q}$, it follows that $H_{\mathcal{W V}}(Q \times I) \subseteq W_{Q}$, and that $f_{\mathcal{V}}(Q)=H_{\mathcal{W V}}(Q \times\{1\}) \subseteq W_{Q}$.

REMARK 2.8. Let $\mathcal{V}$ be a disjoint covering. For every proximate net $\left(f_{\mathcal{U}}\right): X \rightarrow Y$ we can defne a proximate net $\left(f_{\mathcal{U}}^{\prime}\right): X \rightarrow Y$ by putting

$$
f_{\mathcal{U}}^{\prime}=\left\{\begin{array}{l}
f_{\mathcal{U}}, \quad \text { if } \mathcal{U} \prec \mathcal{V} \\
f_{\mathcal{U} \cap \mathcal{V}}, \text { if not } \mathcal{U} \prec \mathcal{V} .
\end{array}\right.
$$


Since $f_{\mathcal{U} \cap \mathcal{V}}$ is $\mathcal{U} \cap \mathcal{V}$ continuous, it follows that it is $\mathcal{U}$ continuous. If $\mathcal{U}^{\prime} \prec \mathcal{U}$, then $\mathcal{U}^{\prime} \cap \mathcal{V} \prec \mathcal{U} \cap \mathcal{V}$, and since $f_{\mathcal{U}}=f_{\mathcal{U} \cap \mathcal{V}}$ and $f_{\mathcal{U}^{\prime}}=f_{\mathcal{U}^{\prime} \cap \mathcal{V}}$ are $\mathcal{U} \cap \mathcal{V}$-homotopic, it follows that they are $\mathcal{U}$-homotopic.

Then, $\left(f^{\prime} \mathcal{U}\right)$ is a proximate net, and $\left(f_{\mathcal{U}}\right)$ and $\left(f^{\prime} \mathcal{U}\right)$ are homotopic.

Since restriction of $\mathcal{U}$-continuous function is $\mathcal{U}$-continuous, and $f_{\mathcal{U}}(Q) \subseteq$ $W_{Q}$ for all coverings $\mathcal{U}$, it follows that the restriction $\left(f^{\prime} \mathcal{U}\right): Q \rightarrow W_{Q}$ is a proximate net.

Corollary 2.9. Let $\left(f_{\mathcal{V}}\right): X \rightarrow Y$ be a proximate net, and $\mathcal{W}$ be a covering consisting of disjoint open sets. Then, for each component $Q \in \square X$, there exists $W_{Q} \in \mathcal{W}$ such that for every $\mathcal{V} \prec \mathcal{W}, f_{\mathcal{V}}(Q) \subseteq W_{Q}$ (and specially $\left.f_{\mathcal{W}}(Q) \subseteq W_{Q}\right)$.

\section{Compact Metric CASE}

In the case of compact metric spaces it is enough to consider proximate sequences $\left(f_{n}\right): X \rightarrow Y$, indexed by the set of natural numbers, [13].

A sequence of finite coverings, $\mathcal{V}_{1} \succ \mathcal{V}_{2} \succ \ldots$ of a space such that for any covering $\mathcal{V}$, there exists $n \in \mathbb{N}$, such that $\mathcal{V} \succ \mathcal{V}_{n}$, we call a cofinal sequence of finite coverings.

Definition 3.1. The sequence $\left(f_{n}\right)$ of functions $f_{n}: X \rightarrow Y$ is a proximate sequence from $X$ to $Y$, if there exists a cofinal sequence of finite coverings, $\mathcal{V}_{1} \succ \mathcal{V}_{2} \succ \ldots$ of $Y$, such that for each natural number $n$ the function $f_{n}: X \rightarrow Y$ is a $\mathcal{V}_{n}$ - continuous function, and if $m \geq n$ then $f_{n}$ and $f_{m}$ are homotopic as $\mathcal{V}_{n}$-continuous functions. In this case we say that $\left(f_{n}\right)$ is a proximate sequence over $\left(\mathcal{V}_{n}\right)$.

Two proximate sequences $\left(f_{n}\right)$ and $\left(f_{n}^{\prime}\right): X \rightarrow Y$ are homotopic if $f_{n}$ and $f_{n}^{\prime}$ are $\mathcal{V}_{n}$-homotopic, for all $n \in \mathbb{N}$.

This is an equivalence relation.

Let $\left(f_{n}\right): X \rightarrow Y$ be a proximate sequence over $\left(\mathcal{V}_{n}\right)$ and $\left(g_{k}\right): Y \rightarrow Z$ be a proximate sequence over $\left(\mathcal{W}_{k}\right)$. For a covering $\mathcal{W}_{k}$ of $Z$, there exists a covering $\mathcal{V}_{n_{k}}$ of $Y$ such that $g_{k}\left(\mathcal{V}_{n_{k}}\right) \prec \mathcal{W}_{k}$. Then, the composition is a proximate sequence $\left(h_{k}\right)=\left(g_{k} f_{n_{k}}\right): X \rightarrow Z$.

Spaces and homotopy classes of proximate sequences form the category whose isomorphisms induces classification of compact metric spaces which coincide with shape classification, i.e., isomorphic spaces in this category have the same shape.

THEOREM 3.2. If $X$ and $Y$ are compact metric spaces, then any proximate sequence $\left(f_{n}\right): X \rightarrow Y$ induces a function

$$
\left(f_{n}\right)_{\#}: \square X \rightarrow \square Y .
$$

Proof. Let $\left(f_{n}\right): X \rightarrow Y$ be a proximate sequences over covering $\left(\mathcal{V}_{n}\right)$ and let $C$ be a component of connectedness of the point $x \in X$. There exists 
a point of accumulation $y \in Y$, of the sequence $\left(f_{n}(x)\right)$, i.e. there exists a subsequence $\left(f_{n_{i}}\right)$ such that $f_{n_{i}}(x) \rightarrow y$.

Suppose $y \in D$, where $D$ is the component of connectedness of $Y$.

We will show that $D$ does not depend on the choice of the accumulation point.

Suppose the contrary, there exists another point of accumulation $z \in E \neq$ $D, E$ a component of $Y$, such that $f_{n_{j}}(x) \rightarrow z$.

Then there exist an open disjoint sets $V$ and $W$ such that $D \subseteq V, E \subseteq$ $W$, and $\mathcal{W}=\{V, W\}$ is a covering of $Y$.

There exists $n_{0}$ such that $f_{n_{0}}$ is $\mathcal{W}$-continuous functions and for $n \geq$ $n_{0}, f_{n}$ and $f_{n_{0}}$ are homotopic as $\mathcal{V}_{n_{0}}$-continuous functions, and it follows as $\mathcal{W}$-continuous functions. Then if $f_{n_{0}}(x) \in V$ it follows that $f_{n}(x) \in V$ for all $n \geq n_{0}$. And this is a contradiction. (the same contradiction is obtained if we suppose $\left.f_{n_{0}}(x) \in W\right)$.

We will show that $D$ does not depend on the choice of $x \in C$.

If $x^{\prime} \in C$ and the component $E$ of $Y$ is chosen, in the same way like the component $D$ is chosen for $x$, then there exist two open disjoint sets $V$ and $W$ such that $V \cup W=Y$, and $D \subseteq V, E \subseteq W$. Put $\mathcal{W}=\{V, W\}$. By Theorem 2.3, there exists $n$ such that $f_{n}(C) \subseteq V$ and $f_{n}(C) \subseteq W$ which is a contradiction.

It follows that the function $\left(f_{n}\right)_{\#}: \square X \rightarrow \square Y$ defined by $\left(f_{n}\right)_{\#}(C)=$ $D$ is well defined.

We omit the proof of the following theorem since is similar to the corresponding theorem of the next section.

THEOREM 3.3. Let $\left(f_{n}\right),\left(g_{n}\right): X \rightarrow Y$ be two proximate sequences. If $\left(f_{n}\right) \sim\left(g_{n}\right)$, then the induced functions are equal, i.e.,

$$
\left(f_{n}\right)_{\#}=\left(g_{n}\right)_{\#}: \square X \rightarrow \square Y
$$

\section{NON COMPACT CASE}

Let $X$ and $Y$ be locally compact metric spaces with compact space of quasicomponents.

THEOREM 4.1. If $X$ and $Y$ are topological spaces with compact space of quasicomponents $Q Y$, then any proximate net $\left(f_{\mathcal{V}}\right): X \rightarrow Y$ induces a continuous function

$$
\left(f_{\mathcal{V}}\right)_{\#}: Q X \rightarrow Q Y
$$

Proof. If $\left(f_{\mathcal{V}}\right): X \rightarrow Y$ is a proximate net, and $Q$ is a quasicomponent of $X$, then for a disjoint covering $\mathcal{W}$ of $Y$, there exists $W_{Q} \in \mathcal{W}$ such that $f_{\mathcal{W}}(Q) \subseteq W_{Q}$. Moreover if $\mathcal{W}^{\prime}$ is another disjoint covering of $Y$ such that $\mathcal{W} \succ \mathcal{W}^{\prime}$, and if $f_{\mathcal{W}^{\prime}}(Q) \subseteq W_{Q}^{\prime}$ then $W_{Q}^{\prime} \subseteq W_{Q}$. 
To prove the last statement we denote by $F_{\mathcal{W} \mathcal{W}^{\prime}}$ the homotopy connecting $f_{\mathcal{W}}$ and $f_{\mathcal{W}^{\prime}}$. Since $F_{\mathcal{W} \mathcal{W}^{\prime}}$ is st $\mathcal{W}$-continuous there exists $W_{1} \in \mathcal{W}$ such that $F_{\mathcal{W} \mathcal{W}^{\prime}}(Q \times I) \subseteq \operatorname{st} W_{1}$.

Since $\mathcal{W}$ is disjoint st $\mathcal{W}_{1}=\mathcal{W}_{1}$, and since $F_{\mathcal{W} \mathcal{W}^{\prime}}(Q \times\{0\})=f_{\mathcal{W}}(Q) \subseteq W_{Q}$ we obtain $W_{1}=W_{Q}$. Then from $f_{\mathcal{W}^{\prime}}(Q)=F_{\mathcal{W} \mathcal{W}^{\prime}}(Q \times\{1\}) \subseteq W_{Q}^{\prime}$ it follows that $W_{Q}^{\prime} \subseteq W_{Q}$.

Notice that there always exists a covering which is finer than a finite number of coverings. From the previous statement it follows that for a fixed $Q$, the intersections of a finite number of sets $Q W_{Q}=\left\{A \mid A \in Q Y, A \subseteq W_{Q}\right\}$ is not empty.

The set $Q W_{Q}=\left\{A \mid A \in Q Y, A \subseteq W_{Q}\right\}$ is closed in $Q Y$. Since $Q Y$ is compact it follows that the intersection of all $Q W_{Q}$, where $W_{Q}$ is taken from a disjoint covering $\mathcal{W}$, is not empty and consists of one quasicomponent $T$. We put $\left(f_{\mathcal{W}}\right)_{\#}(Q)=T$.

To prove the continuity of $f_{\#}$, let

$$
f_{\#}(S)=T, \quad\left(f_{\mathcal{V}}\right): X \rightarrow Y
$$

be a proximate net and $G, G \supseteq T$, be a clopen subset of $Y$. Then $Q G=$ $\{A \mid A \in Q Y, A \subseteq G\}$ is a basis neighborhood of $T$ in $Q Y$. There exists a $\mathcal{V} \in \operatorname{cov} Y$ that refines the covering $\{G, Y \backslash G\}$ of $Y$. Since the function $f_{\mathcal{V}}$ is $\mathcal{V}$-continuous, it is $\{G, Y \backslash G\}$-continuous, also.

Now, we will show that the set

$$
F=\left\{x \mid x \in X, f_{\mathcal{V}}(x) \in G\right\}
$$

is clopen in $X$. Suppose $x \in F$. Since the function $f_{\mathcal{V}}$ is $\{G, Y \backslash G\}$-continuous and $f_{\mathcal{V}}(x) \in G$, it follows that there exists a neighborhood $U$ of $x$ such that $f_{\mathcal{V}}(U) \subseteq G$. We obtained that $U \subseteq F$, so $F$ is open. Similarly, $X \backslash F=$ $\left\{x \mid x \in X, f_{\mathcal{V}}(x) \in Y \backslash G\right\}$ is open, i.e., $F$ is closed.

Since $S \subseteq F, Q F$ is a neighborhood of $S$ in $Q X$. If $S^{\prime} \in Q F$, then $f_{\{G, Y \backslash G\}}\left(S^{\prime}\right) \subseteq G$, and from the construction of $f_{\#}$ it follows that $f_{\#}\left(S^{\prime}\right) \in$ $Q G$. We obtained $f_{\#}(Q F) \subseteq Q G$, i.e., $f_{\#}$ is continuous.

ThEOREM 4.2. Let $\left(f_{\mathcal{V}}\right),\left(g_{\mathcal{V}}\right): X \rightarrow Y$ are homotopic proximate nets $\left(f_{\mathcal{V}}\right) \sim\left(g_{\mathcal{V}}\right)$. Then, for the induced maps holds:

$$
\left(f_{\mathcal{V}}\right)_{\#}=(g \mathcal{V})_{\#}
$$

Proof. Let $\left(H_{\mathcal{V}}\right): X \times I \rightarrow Y$ be a proximate net, i.e., $H_{\mathcal{V}}: X \times I \rightarrow Y$ is a homotopy connecting $\mathcal{V}$ continuous functions $f_{\mathcal{V}}, g_{\mathcal{V}}: X \rightarrow Y$.

Let $Q$ be a quasicomponent of $X$ such that $\left(f_{\mathcal{V}}\right)_{\#}(Q)=T,\left(g_{\mathcal{V}}\right)_{\#}(Q)=R$ and $T \neq R$. Then there exist an open disjoint sets $V$ and $W$ such that $T \subseteq V, \quad R \subseteq W$, and $\mathcal{W}=\{V, W\}$ is a covering of $Y . H_{\mathcal{W}}: X \times I \rightarrow Y$ is a st $\mathcal{W}$-continuous, and since st $\mathcal{W}=\mathcal{W},\left(H_{\mathcal{W}}\right): X \times I \rightarrow Y$ is $\mathcal{W}$-continuous.

Let $x$ be an arbitrary point from the quasicomponent $Q$. Then $H_{\mathcal{W}}((x, 0))=f_{\mathcal{W}}(x) \in V$, while $H_{\mathcal{W}}((x, 1))=g_{\mathcal{W}}(x) \in W$. This is a 
contradiction, since by Theorem 2.3, $H_{\mathcal{W}}(Q \times I) \subseteq V$ or $H_{\mathcal{W}}(Q \times I) \subseteq W$. So, $T=R$, i.e., for the induced maps holds: $\left(f_{\mathcal{V}}\right)_{\#}=\left(g_{\mathcal{V}}\right)_{\#}$.

The last theorem is the main result, an analogue of Borsuks theorem, in the case of non compact spaces. It is a consequence of Theorems 4.1, 4.2 and 2.7 .

THEOREM 4.3. Suppose $X$ and $Y$ are locally compact metric spaces with compact spaces of quasicomponents $Q X$ and $Q Y$.

If a shape morphism $f: X \rightarrow Y$ is presented by a proximate net $\left(f_{\mathcal{V}}\right)$ : $X \rightarrow Y$, then there exists a unique mapping $\left(f_{\mathcal{V}}\right)_{\#}: Q X \rightarrow Q Y$, such that, if $\left(f_{\mathcal{V}}\right),\left(g_{v}\right): X \rightarrow Y$ are homotopic proximate nets such that $\left(f_{\mathcal{V}}\right) \sim\left(g_{v}\right)$, i.e., they define the same shape morphism, then $\left(f_{\mathcal{V}}\right)_{\#}=\left(g_{\mathcal{V}}\right)_{\#}$.

Moreover, the restriction of $f$ to any clopen set $W$ containing $\left(f_{\mathcal{V}}\right)_{\#}(Q)$, presented by the restriction of proximate net $\left(f_{\mathcal{V}}\right): Q \rightarrow W$ is also a shape morphism.

\section{REFERENCES}

[1] Y. Akaike, K. Sakai, Describing the proper n-shape category by using non-continuous functions, Glas. Mat. Ser. III 33 (1998), no. 2, 299-321.

[2] B. J. Ball, Shapes of saturated subsets of compacta, Colloq. Math. 29 (1974) 241-246.

[3] B. J. Ball, Quasicompactifications and shape theory, Pacific J. Math. 84 (1979), 251259.

[4] B. J. Ball, Partitioning shape-equivalent spaces, Bull. Acad. Pol. Math. 29 (1981), 491-497.

[5] K. Borsuk, Theory of shape, Polish Scientific publishers, Warszawa, 1975.

[6] R. Engelking, General Topology, Monografie Matematiczne, Warszawa, 1977.

[7] R. W. Kieboom, An intrinsic characterization of the shape of paracompacta by menas of non-continuous single-valued maps, Bull. Belg. Math. Soc. Simon Stevin 1 (1994), 701-711.

[8] Y. Kodama, Decomposition spaces and shape in the sense of Fox, Fund. Math. 97 (1977), 199-208.

[9] M. A. Moron, N-compactness and shape, Proc. Amer. Math. Soc. 113 (1991), 545-550.

[10] M. A. Moron, On the Wallman-Frink compactification of 0-dimensional spaces and shape, Arch. Math. (Basel) 58 (1992), 294-300.

[11] M. A. Moron, F. R. Ruiz del Portal and J. M. R. Sanjurjo, Shape invariance of $\mathbb{N}$ compactifications, Topology Appl., 56 (1994) 63-71.

[12] J. M. R. Sanjurjo, On a theorem of B. J. Ball, Bull. Polish Acad. Sci. Math. 33 (1985), $177-180$.

[13] N. Shekutkovski, Intrinsic definition of strong shape for compact metric spaces, Topology Proc. 39 (2012), 27-39.

[14] N. Shekutkovski and G. Markoski, Equivalence of the inverse system approach and the intrinsic approach to proper shape, God. Zb. Inst. Mat. Prir.-Mat. Fak. Univ. Kiril Metodij Skopje 41 (2008) 43-56.

[15] N. Shekutkovski and G. Markoski, Ends and quasicomponents, Cent. Eur. J. Math., 8 (2010), 1009-1015. 
N. Shekutkovski

Institute of Mathematics

Faculty of Natural Sciences and Mathematics

Sts. Cyril and Methodius University

1000 Skopje

Republic of Macedonia

E-mail: nikita@pmf.ukim.mk

T. Atanasova-Pachemska

University "Goce Delchev" - Shtip

Faculty of Informatics

2000 Shtip

Republic of Macedonia

E-mail: tatjana.pacemska@ugd.edu.mk

G. Markoski

Institute of Mathematics

Faculty of Natural Sciences and Mathematics

Sts. Cyril and Methodius University

1000 Skopje

Republic of Macedonia

E-mail: gorgim@pmf .ukim.mk

Received: 26.4.2011.

Revised: 9.9.2011. 